\title{
Modèle exploratoire de la dissonance et de l'harmonie ludo-narrative dans les jeux d'évasion
}

An exploratory model of ludo-narrative dissonance and harmony in escape games

\section{Antoine Taly, Annelyse Nugue et Michael Freudenthal}

\section{OpenEdition}

\section{Journals}

Édition électronique

URL : https://journals.openedition.org/sdj/3718

DOI : $10.4000 /$ sdj.3718

ISSN : 2269-2657

Éditeur

Laboratoire EXPERICE - Centre de Recherche Interuniversitaire Expérience Ressources Culturelles Education

Référence électronique

Antoine Taly, Annelyse Nugue et Michael Freudenthal, « Modèle exploratoire de la dissonance et de I'harmonie ludo-narrative dans les jeux d'évasion», Sciences du jeu [En ligne], 16 | 2021, mis en ligne le 08 novembre 2021, consulté le 26 novembre 2021. URL : http://journals.openedition.org/sdj/3718 ; DOI : https://doi.org/10.4000/sdj.3718

Ce document a été généré automatiquement le 26 novembre 2021.

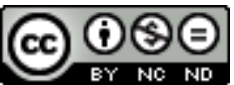

La revue Sciences du jeu est mise à disposition selon les termes de la Licence Creative Commons Attribution - Pas d'Utilisation Commerciale - Pas de Modification 4.0 International. 


\title{
Modèle exploratoire de la dissonance et de l'harmonie ludo- narrative dans les jeux d'évasion
}

An exploratory model of ludo-narrative dissonance and harmony in escape games

\author{
Antoine Taly, Annelyse Nugue et Michael Freudenthal
}

1 Le principe initial des jeux d'évasion (JE) appelés le plus souvent Escape Games est simple : il faut sortir d'une pièce dans laquelle on est enfermé1. Que la pièce soit réelle, dans le cas des jeux grandeur nature, ou virtuelle, dans le cas des jeux sur table ou vidéo, ne change pas ce principe. Une des propriétés des JE rapidement mise en avant est leur capacité à immerger les joueurs dans un autre univers (Wiemker et al., 2005). Ainsi, dans un JE grandeur nature, les joueurs sont enfermés dans une salle décorée en donjon médiéval, en sous-marin ou encore en bureau du FBI, et deviennent des explorateurs, des détectives, ou encore des voleurs. Il apparaît dès lors important de questionner cette notion d'immersion.

2 Néanmoins, la notion d'immersion paraît souvent floue tant dans la communication commerciale que dans la recherche (Bonenfant et Philippette, 2018). Une façon d'analyser l'immersion dans les JE est de prendre explicitement en compte son articulation avec l'engagement (Blumenthal et Jensen, 2019). L'immersion peut alors être vue comme un processus progressif, qui se nourrit des différentes formes d'engagements. Cette approche sera utilisée pour tester l'utilisation de nouveaux outils conceptuels pour étudier le potentiel d'immersion des JE via l'engagement.

3 Par ailleurs, en nous penchant sur l'historique et l'évolution des JE, nous notons un développement marquant de la narration. Cela pourrait renforcer l'immersion dans l'univers fictif (Trépanier-Jobin et Couturier, 2018), ou constituer une nouvelle voie pour entrer dans l'immersion (Blumenthal, 2020). Cependant, le développement de la narration pose aussi la question de la cohérence avec les mécaniques ludiques présentes dès l'origine des JE. Nous nous proposons donc d'analyser la dimension ludo- 
narrative des JE et l'influence potentielle de la cohérence de la dimension narrative avec les mécaniques ludiques sur l'immersion.

\section{Quelques éléments sur l'origine des jeux d'évasion}

4 L'origine des JE n'est pas clairement établie, mais on trouve des éléments à partir desquels les JE vont être construits ou pensés dès les années 1980 dans le domaine du jeu vidéo (Stasiak, 2016). En 2007, le concept est transposé de manière tangible, avec le Real escape game, qui est introduit dans des bars ou des clubs de Kyoto aménagés spécifiquement pour l'occasion. Sans aucune histoire ni thème, le jeu propose à une équipe de cinq à six joueurs enfermée dans une pièce comportant des inscriptions étranges et des objets insolites de surmonter des épreuves (principalement des énigmes) pour pouvoir s'échapper de la salle en moins d'une heure. Les participants disposent d'une feuille de papier qu'ils doivent remplir avec les réponses aux défis. À chaque réponse trouvée, un organisateur du JE (appelé maître du jeu) la vérifie et, si elle est correcte, donne un objet ou un indice permettant de résoudre le défi suivant. À la suite du succès de cette première salle, de nombreux JE ont été créés en Asie d'abord, puis en Europe, dès 2011 à Budapest, et enfin en Angleterre puis en France en 2013. Simultanément, le JE atteint les États- Unis et le Canada (French et Marmor Shaw, 2015).

\section{Evolution des jeux d'évasion}

5 Les JE créés en 2021 étant bien différents des tout premiers, il est légitime de se poser la question de leur évolution. Scott Nicholson propose un modèle générationnel qui se base sur la complexité du récit (Nicholson, 2016):

- Les salles centrées sur les défis (génération 1) : la salle consiste en une succession de défis sans thème, décor ni histoire en particulier.

- Les salles thématiques (génération 2): la salle est ancrée dans un genre et un cadre spécifique (par exemple l'univers de la piraterie, ou le genre détective) que le décor et les défis reflètent.

- Les salles narratives (génération 3): c'est une salle thématique à laquelle une histoire comportant un début, des péripéties et une fin a été intégrée aux défis. Les joueurs ont également un rôle défini dans cette histoire (par exemple, ce sont des espions qui doivent récupérer des documents secrets).

- Les salles hypernarratives (génération 4): il s'agit d'une salle narrative dans laquelle l'histoire est influencée par les choix des joueurs. Plusieurs embranchements sont disponibles, et il est impossible de voir toute l'histoire en une seule partie. Les joueurs doivent parfois faire de l'improvisation théâtrale et interagir avec des personnages non joueurs (PNJ) incarnés par des acteurs ou le maître du jeu.

6 Si des JE de génération 1 ou 2 sont encore créés à l'heure actuelle, on constate cependant que de plus en plus de JE modernes mettent en avant la narration, l'univers et les personnages. Ces éléments permettent d'offrir aux joueurs une expérience immersive parfois très proche du jeu de rôle Grandeur Nature (GN). 


\section{Définir le jeu d'évasion}

7 Pour établir une définition, Annelyse Nugue s'est basée sur les nombreux usages du JE et de ses différentes formes. Elle s'est de même appuyée sur les définitions proposées par quatre concepteurs et conceptrices de JE afin de les comparer et de construire la définition suivante: "L'escape game est un jeu dans lequel une équipe de joueurs interagit physiquement avec les éléments contenus dans un espace thématique et immersif. Les joueurs coopèrent pour découvrir et surmonter des défis afin d'atteindre un objectif dans un temps imparti » (Nugue, 2019, p. 15).

8 Cette définition exclut les jeux qui ne sont pas thématiques, ce qui correspond à la tendance actuelle : en 2015 , seules $13 \%$ des salles dans le monde n'avaient pas de thème défini et restaient donc dans leur forme initiale basées uniquement sur les énigmes (Nicholson, 2015). Elle permet ainsi de nous concentrer sur les cas qui nous concernent, ceux pour lesquels la narration est importante, et donc permettent d'étudier l'impact potentiel de celle-ci sur l'immersion.

\section{La question des relations ludo-narratives}

\section{Approcher l'immersion du joueur à travers l'analyse de la cohérence du jeu.}

9 Malgré l'importance que prend l'immersion dans les jeux d'évasion où la narration est devenue centrale, il n'existe pas de modèle adapté pour l'analyser dans les JE. Notre objectif est de présenter un tel modèle d'immersion adapté aux JE. Nous nous appuierons sur les travaux de Blumenthal et Jensen (2019) qui proposent d'analyser l'immersion comme un processus progressif, qui se nourrit des différentes formes d'engagements. Cela nous conduit à nous pencher sur l'engagement comme un préalable à l'immersion (Arsenault et Picard, 2008; Bonenfant et Philippette, 2018). Ainsi, tout élément qui agit sur l'engagement agit aussi, indirectement, sur l'immersion. Cette approche permet ainsi d'analyser les facteurs qui peuvent influer sur l'immersion (Blumenthal, 2020), et permet donc d'analyser plus finement l'immersion sans la traiter comme un processus essentiellement binaire (Hou et Chou, 2012).

10 L'engagement dans l'activité ludique implique d'explorer une réalité seconde (Brougère, 2005), de franchir le cercle magique (Huizinga, 1951; Salen et Zimmerman, 2003). Au niveau de l'individu, on peut rapprocher la démarche de la suspension volontaire d'incrédulité, qui correspond à « l'inclination qu'ont les lecteurs à ignorer les incohérences de l'histoire en raison de leur volonté de construire un récit cohérent» (Karhulahti, 2012, p. 1). Différentes formes d'engagement peuvent alors entrer en compte (Caïra, 2018).

11 Par ailleurs, de nombreux paramètres pourraient jouer sur l'engagement et donc l'immersion, en particulier la structure du jeu. En effet, de nombreux aspects peuvent influer sur la suspension d'incrédulité, ainsi Karhulahti (2012) répertorie : L'histoire, les aspects techniques, les personnages. De plus Ermi et Mäyrä (2005, p. 95) soulignent qu'elle permet «de s'incarner dans un monde irréaliste si celui-ci reste cohérent». 
Enfin, il a été observé que l'immersion est dépendante de la cohérence de l'expérience ludique et de sa réception collective (Kapp, 2013).

\section{Les relations ludo-narratives}

12 Puisque le développement de la narration est un des axes d'évolution des JE, nous nous sommes posé la question de son intégration aux mécaniques ludiques. L'interactivité a souvent été désignée comme étant un obstacle à l'intégration réussie du récit dans un jeu, puisque d'une part le récit doit rester inchangé pour préserver sa pertinence et sa richesse, et d'autre part l'interactivité a pour objectif de donner au joueur un moyen d'agir, de faire des choix et de modifier les éléments (Adams, 1999). La question est donc de savoir comment combiner récit et interactivité pour une meilleure expérience de jeu, et donc une immersion. Puisqu'il y a peu d'éléments dans la littérature sur les JE, nous nous appuierons sur la littérature plus fournie sur les jeux vidéo (Koenitz, 2018).

Le terme de dissonance ludo-narrative a été proposé par Hocking (2009), qui remarque que la structure ludique du jeu vidéo Bioshock (2K Games, 2007) est en contradiction avec sa structure narrative. Le jeu propose au joueur un contrat ludique qui est de pouvoir choisir entre une approche égoïste (privilégier son intérêt personnel en récoltant des PNJ appelés Petites Sœurs, ce qui entraîne leur mort mais octroie au joueur davantage de puissance) ou altruiste (sauver les Petites Sœurs au détriment de la puissance). Pourtant, le contrat narratif force le joueur à adopter une approche altruiste en lui donnant comme missions obligatoires d'aider la famille d'un autre PNJ et de sauver certaines Petite Sœurs. D'un côté, les actions que le joueur a effectuées l'ont amené à considérer son avatar comme étant égoïste. De l'autre côté, le récit l'amène à considérer son avatar comme étant altruiste. Cette incohérence a conduit Hocking à nommer ce sentiment de paradoxe une dissonance ludo-narrative (DLN).

Pour analyser la DLN, nous pouvons utiliser le modèle d'analyse ludo-narrative proposé par Sébastien Genvo (2020). Ce modèle permet en particulier de lier la protohistoire, telle que conçue par les concepteurs et maîtres de cérémonie, et l'histoire, qui résulte de l'action et de l'interprétation des joueurs, via les boucles de la jouabilité (gameplay). Ce modèle présente ainsi l'expérience ludique comme dépendante de la communication qui s'opère entre le joueur et le jeu. Les éléments de médiation qui sont mis en place pour réguler l'expérience ludique apparaissent donc comme des facteurs cruciaux de l'analyse des relations ludo-narratives (Grandjean, 2020).

Nous suggérons également de rapprocher la notion de dissonance ludo-narrative de la théorie de la dissonance cognitive établie par Festinger (1957). Il définit une connaissance comme étant une croyance, une opinion ou un savoir que l'on a à propos de l'environnement, de soi-même ou de son comportement. Festinger détermine trois types de relations pouvant exister entre deux connaissances : la dissonance, l'harmonie et l'indépendance.

En nous basant sur l'approche de Pynenburg (2012), nous définissons l'harmonie ludonarrative comme une relation synergique entre les connaissances que le joueur acquiert par la jouabilité et celles qu'il obtient par le récit d'un jeu. Nous définissons l'indépendance ludo-narrative comme l'absence de toute relation positive ou négative entre des connaissances que le joueur acquiert par le récit d'un jeu et sa jouabilité. À la suite de Sébastien Genvo, nous choisissons d'analyser les relations ludo-narratives en considérant la totalité de l'expérience ludique, du jeu au joueur. Nous suggérons de le 
faire avec les deux modèles que nous allons présenter et adapter, celui d'Aarseth (2012) et celui de Caïra (2018).

\section{Le modèle d'analyse narrative d'Aarseth : identification des éléments du jeu}

Le modèle d'analyse narrative d'Aarseth (2012) identifie les dénominateurs communs à tous les jeux permettant de comprendre la relation entre le récit et le jeu. Il repose sur quatre éléments fondamentaux et indépendants que l'on retrouve dans chaque histoire et dans chaque jeu :

- Le monde (game world), définit comme «une structure physique ou pseudo-physique (virtuelle) qui est clairement délimitée et qui peut être décrite géométriquement ou de façon topologique » (Aarseth, 2012, p. 4). En cela, il s'oppose au monde fictionnel qui n'existe que dans l'imagination.

- Les objets. Aarseth les classe selon leur degré de malléabilité. Dans un jeu vidéo, une interaction avec un objet ne peut avoir lieu que si celle-ci a été intégrée au code du jeu. Dans un JE, le fait que les objets soient physiques donne au joueur une grande liberté. Rien n'empêche les joueurs de détruire les objets de la salle ou de forcer un cadenas pour ouvrir un coffre, si ce n'est les cadres sociaux dans lesquels ils se trouvent (ludiques, sociétaux, légaux, etc.).

- Les agents. Dans un jeu vidéo, les personnages peuvent être classés en deux catégories : ceux contrôlés par un joueur (Personnage Joueur ou «PJ ») et les autres (Personnage Non-Joueur ou «PNJ»). Dans le cas des JE, les PNJ sont incarnés le plus souvent par des acteurs. Le maître du jeu est parfois un PNJ.

- Les évènements. Nous proposons que dans le cadre d'un JE, les défis soient considérés comme des évènements. Plusieurs organisations de défis récurrentes dans les JE ont été identifiées par Nicholson (2015) et Clare et Choudhury (2016).

\section{Le modèle MEDIAL : les dimensions de l'engagement ludique}

Le sentiment d'immersion en jeu vidéo a été étudié surtout du point de vue de l'état de concentration optimal (flow) théorisé par Csíkszentmihályi (Caïra, 2018), pourtant ce n'est pas le seul état d'engagement qui existe, en particulier dans les JE. Les modèles du " flow» se concentrent en effet sur sa dimension individuelle, or les JE proposent aux visiteurs à la fois une opportunité d'interagir avec d'autres personnes et une immersion dans un autre univers (Williams et Mascioni, 2014). L'étude empirique de Kolar et Čater (2018) identifie des éléments qui influent sur cette immersion de groupe : l'équilibre entre les compétences du groupe et la difficulté des épreuves, ainsi que la thématisation et la narration. La notion d'immersion de groupe ( group flow») a ainsi été introduite pour les JE (Kolar et Čater, 2018). Cette étude, néanmoins, ne nous semble pas détailler suffisamment les caractéristiques des JE.

Nous nous proposons donc ici de faire appel au modèle MEDIAL développé par Caïra (2018) et qui inclut l'aspect collectif. Les dimensions proposées par le modèle MEDIAL s'appliquent particulièrement bien aux $\mathrm{JE}$, ce qui en fait une bonne base pour analyser l'engagement, notamment dans la dimension collective déjà évoquée. 
Il est composé de six dimensions d'engagement dans l'activité ludique :

- Moteur : "l'intérêt porté au système de règles, à la "mécanique" logico-mathématique ou physique qui régit le jeu » mais aussi la possibilité « d'explorer les potentialités et les limites de ce système » (\$23). Dans le cadre des JE, on retrouve les énigmes ainsi que les tentatives pour les contourner.

- Épreuve : la possibilité de mettre en œuvre ses compétences physiques et cognitives. Pour les JE la dimension coopérative entraîne que les compétences sont celles des membres du groupe et sa capacité à utiliser la complémentarité de ses membres.

- Diégèse : c'est-à-dire l'univers (fictionnel) spatio-temporel désigné par le récit. Caïra note que « de nombreux développeurs de jeux ont misé sur la thématisation, sur le worldbuilding et sur une forte promesse d'immersion pour renforcer l'attrait de leurs productions » (\$25). On retrouve ici la thématisation apparue rapidement dans le développement des JE.

- Intrigue : « une tension narrative sur les événements au sein de leur diégèse » (\$26). Dans le cadre des JE on retrouve les potentialités offertes par les JE narratif ou hyper-narratif et le rôle des PNJ.

- Autrui : «Créer des interactions sociales, voire nouer des relations, peut être un objectif en soi ou un simple effet induit de l'engagement ludique » (\$27). Les JE étant coopératifs, cette dimension est très présente notamment parce que la communication est un facteur clef de la progression de l'équipe dans le jeu.

- Ludologie : «les joueurs passionnés développent une pensée réflexive et de plus en plus érudite» (\$28). Dans le cadre des JE cette dimension est explorée par exemple dans des énigmes qui reposent sur des jeux connus comme le Rubik's Cube.

21 Nous notons que certaines des six dimensions d'engagement sont directement liées à la notion de lien ludo-narratif. En effet, le Récit selon Genette (1972), et comme nous l'utilisons ici, comprend la Diégèse et l'Intrigue. En outre le pôle ludique correspond bien aux dimensions moteur et épreuve. Par ailleurs, on peut décrire l'évolution des JE présentée plus haut en s'appuyant sur ces dimensions de l'engagement. Les dimensions Autrui, Épreuve et Moteur étaient présentes dès la première génération de JE. La deuxième génération ayant vu l'apparition de la Diégèse et la troisième de l'Intrigue. Par contre, il convient de noter que la dimension d'engagement "Ludologique » est potentiellement en désaccord avec la notion d'immersion. Puisque nous utilisons les dimensions de l'engagement pour approcher l'immersion, cette dimension sera écartée de notre analyse.

Dans le modèle MEDIAL, les différentes dimensions sont représentées par des flèches qui s'écartent les unes des autres. C'est une propriété que le modèle MEDIAL hérite du modèle présenté par Calleja (2011). Caïra le présente ainsi : «L'essentiel à retenir, me semble-t-il, est l'idée que ces flèches pointent dans des directions différentes, et qu'un engagement fort dans une des dimensions de l'expérience ludique se fait le plus souvent au détriment des autres dimensions » $\left(\$ 20^{2}\right)$. Autrement dit, les dimensions peuvent être plus ou moins développées et le développement fort d'une dimension peut limiter la possibilité d'explorer les autres dimensions. Nous n'explorons pas davantage l'idée d'incompatibilité entre les dimensions, mais acceptons pleinement qu'il existe une interdépendance entre les dimensions de l'engagement.

Olivier Caïra évoque également deux notions qui s'appliquent à toutes les dimensions, celle de découpage du temps dans l'engagement en-partie et hors-partie et celle de d'intensité d'engagement variant entre sur-engagement et sous-engagement. Dans notre propos, nous ne reviendrons pas sur les variations entre sur et sous engagement, 
bien qu'elles s'appliquent aux JE. Nous notons en revanche que la notion d'engagement en-partie et hors-partie est particulièrement pertinente dans le cas des JE. Ainsi les salles de JE comportent généralement, en plus des salles de jeu, une salle d'accueil confortable où les joueurs peuvent se réunir. Cela permet de remplir le besoin d'interaction, et permet aux joueurs d'essayer d'imaginer l'expérience qu'ils s'apprêtent à vivre, un espace liminal où l'engagement peut débuter, mais aussi se conclure. En effet, en fin de partie, les joueurs retracent l'ensemble des événements survenus pendant le JE, discutent des décisions qu'ils ont prises, des sentiments qu'ils ont ressentis et comment chacun d'entre eux a vécu le JE ; c'est également dans cette salle qu'une photo de groupe peut être prise pour immortaliser le moment (Clare et Choudhury, 2016).

\section{Prolongement du modèle MEDIAL}

24 Une limitation du modèle est que les dimensions y sont vues comme contradictoires, le développement d'une dimension se faisant au détriment des autres. Dans le cas des JE le développement des dimensions Diégèse et Intrigue se ferait donc nécessairement au détriment des dimensions Épreuve et Moteur. Néanmoins, l'analyse s'inspirant de la dissonance cognitive suggère au contraire que les dimensions ne sont pas seulement en contradiction mais peuvent éventuellement être complémentaires. Cette analyse est cohérente avec l'observation faite par Dunne (2014) de la focalisation sur les incohérences quand il s'agit d'étudier des jeux alors qu'une analyse des synergies peut être également féconde.

Nous proposons une extension du modèle MEDIAL pour analyser les relations entre ses différentes dimensions. Chaque élément du modèle pourrait toujours être représenté par une flèche comme dans le modèle original et dans chacune des dimensions, le niveau de développement pourrait délimiter une surface qui représenterait la forme d'engagement du joueur. Enfin, les dimensions pourraient être en harmonie ou en dissonance. Prendre en considération les parties harmonieuses ou dissonantes permettrait de mieux comprendre les dimensions du modèle MEDIAL, leurs interdépendances et leurs intensités. 
Figure 1. Modèle MEDIAL en 2D illustrant harmonie ou dissonance ludo-narrative.
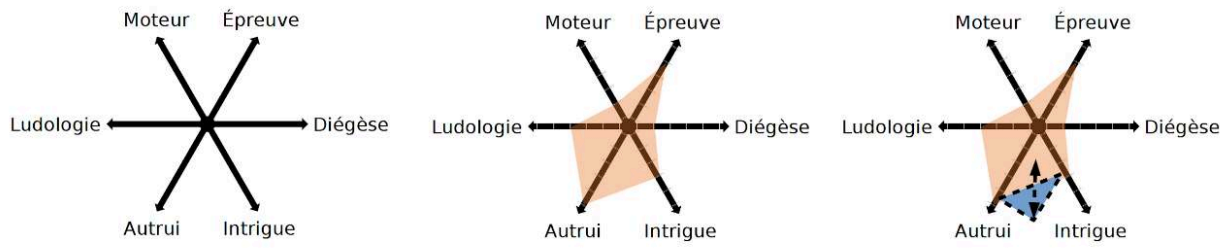

Nous reprenons à gauche dans la figure 1 les dimensions du modèle MEDIAL (Caïra, 2018) dans lequel chaque dimension est représentée par une flèche s'écartant du centre. Nous introduisons ensuite une représentation en "radar», dans laquelle l'exploration de chaque dimension définit une surface qui décrit l'engagement global. Ce radar ne dépend pas d'un score spécifique et a plutôt vocation à être illustratif, pour représenter l'effet des synergies ou antagonismes entre les dimensions. La dernière image suggère ainsi que les différentes dimensions peuvent être en harmonie ou pas et ainsi augmenter ou réduire l'engagement global.

\section{Problématique et observations préliminaires}

\section{Problématique}

Nous avons défini le JE comme un jeu dont l'un des objectifs majeurs est de créer une immersion chez les joueurs. Il n'existe pas de modèle adapté et testé pour l'analyse de l'immersion dans les JE. Atteindre ce but est la finalité de notre travail. Pour ce faire nous chercherons à atteindre des objectifs intermédiaires, à savoir tester d'une part le modèle MEDIAL pour décrire les dimensions de l'engagement du joueur et d'autre part celui d'Aarseth pour identifier les éléments du jeu à analyser.

Bien que de nombreux éléments soient susceptibles de faire sortir les participants de l'immersion, nous avons identifié une tendance forte dans le développement des JE autour des aspects narratifs. Nous avons proposé que les relations entre le récit et la jouabilité d'un jeu peuvent compromettre l'engagement des joueurs et donc sortir le joueur de l'immersion. Cela signifie que si un récit et une jouabilité sont dissonants, l'immersion dans le JE pourrait être compromise. Inversement, l'harmonie et la dissonance ludo-narratives, lorsqu'elles sont utilisées intentionnellement, permettraient de renforcer le message du jeu. 


\section{Cadre des observations préliminaires}

Il est difficile de faire une étude sur la jouabilité et le récit d'un JE pour plusieurs raisons. D'une part, contrairement au jeu vidéo, il est rare de trouver des vidéos de parties de JE en ligne. D'autre part, les critiques de JE ne veulent pas divulguer les solutions des défis et les surprises du JE. Par conséquent, il est ardu de trouver un descriptif détaillé de la jouabilité ou du récit. Nous mobilisons ici des entretiens et des tests réalisés par l'une d'entre nous dans le cadre de sa thèse professionnelle, qui portait sur les relations ludo-narratives dans les escapes games (Nugue, 2019).

Quatre entretiens ont été réalisés avec des concepteurs de jeu qui ont pu parler de leur expérience de conception mais se sont aussi beaucoup référé à leur expérience de jeu : Michael Freudenthal, qui était alors game designer, Fiona Buchou, Alexandre Carturan, Sébastien Decoster.

Afin de comparer ces témoignages à une expérience réelle, Nugue (2019) a également participé et analysé un échantillon de JE :

- Mission Evolvis (Immersia) est un JE sur le thème de la dystopie. L'état de la planète devient critique et une multinationale nommée Evolvis organise un test autour des cinq sens pour sélectionner ceux qui survivront. Les joueurs incarnent des rebelles devant s'infiltrer chez Evolvis pour pirater leur système et obtenir des informations compromettantes. Pour ce faire, ils devront s'inscrire au test d'Evolvis sous couverture et trouver le bon moment pour échapper à l'attention des gardes et voler les données, c'est-à-dire en jouant un rôle face à des acteurs. Ce JE présente un récit riche et développé.

- La Serre (Escape Hunt) est un JE sur un thème mêlant apocalypse et nature. Paris est envahi par la végétation et tout semble converger vers l'étrange laboratoire d'un botaniste. Les joueurs devront alors examiner le laboratoire pour trouver l'origine du problème pour espérer sauver la capitale. Ce JE présente un récit moyennement développé.

- Eclipse (Backlight) est un JE en réalité virtuelle. Les joueurs sont à bord d'une navette spatiale et sont en route pour une mission de sauvetage. La communication a été perdue avec l'équipage de la précédente mission et les joueurs doivent se rendre sur place pour découvrir ce qu'il s'est passé. Ce JE présente un récit moyennement développé en réalité virtuelle.

- Les Maîtres du temps (60 Minutes Escape) a une thématique fantastique. Les joueurs sont envoyés dans le futur pour déjouer les plans d'un dangereux mage. Arrivés dans son repaire, rien ne va se passer comme prévu et ils n'auront plus qu'un objectif, sortir de cet enfer et revenir dans le présent. Ce JE présente un récit peu développé, et est davantage tourné vers la jouabilité.

31 Dans les sections suivantes, nous examinerons les cas récurrents de dissonances et d'harmonie ludo-narratives. Les JE sont décrits à partir des éléments du modèle d'Aarseth : 1) les évènements, 2) le maître du jeu et 3) le monde et les objets. Pour chaque catégorie, des dissonances ludo-narratives ont été identifiées, nous avons essayé d'illustrer chacune par un exemple ainsi que par un contre-exemple d'harmonie ludo-narrative. 


\section{Observations préliminaires : identification des relations ludo-narratives}

\section{La structure générale du JE, les évènements en tant qu'épreuve et récit} p. 28)

\section{Le maître du jeu : à la fois système d'indices et personnage}

Le maître du jeu est la personne responsable de la supervision de l'expérience de jeu, et notamment de la distribution des indices. Nous l'étudierons donc sous l'angle du système d'indices, puis sous l'angle du personnage incarné. 


\section{Les indices explicites} et partielle?

La plupart des JE ont mis en place un système d'aide afin de réguler la difficulté de l'expérience, et donc la dimension épreuve du modèle MEDIAL. Des indications sont données aux joueurs pour les mettre sur la voie de la solution. Nous appelons cette aide les indices explicites, parce que cette information est explicitement indiquée comme étant une aide et vient s'ajouter aux informations déjà présentes dans l'espace de jeu. Les indices explicites s'opposent aux indices implicites: information placée dans l'espace de jeu avant l'arrivée des joueurs. Ceux-ci doivent l'interpréter et parfois les combiner avec d'autres indices implicites afin de résoudre un défi. Il n'est pas indiqué explicitement qu'il s'agit d'un indice ; c'est aux joueurs de le déterminer.

Le système d'indices explicites est dès lors essentiel à l'expérience de jeu. Le maître du jeu ne distribue des indices explicites que de manière ponctuelle, lorsque les joueurs ne parviennent pas à résoudre un défi. De plus, son aide n'est que partielle; bien souvent, les indices sont cryptiques afin de mettre les joueurs sur la voie sans leur donner la réponse. Cette aide a néanmoins aussi un sens du point de vue narratif. Le modèle d'analyse ludo-narrative de Genvo (2020) montre le rôle de l'interprétation de la narration par les joueurs dans l'expérience ludique. Cette interprétation, comme le précise le modèle de Grandjean (2018), est facilitée par une médiation du système de jeu. Dans les JE, la médiation est assurée par le maître du jeu, soit de manière transparente soit en passant par un système numérique ou en incarnant un PNJ.

\section{Indices donnés via un écran}

Il arrive que la manière dont les indices explicites sont donnés n'appartient pas à la diégèse. Nous prenons en exemple le cas du JE Les Maîtres du Temps. Les joueurs y explorent le repère du Mage Noir, dans un univers plutôt fantasy. Les indices explicites sont pourtant affichés par le biais d'un écran. Ainsi, le récit informe le joueur qu'il se trouve dans une crypte en pierre ancienne, tandis que le système d'indices explicites l'informe qu'il se trouve dans une pièce moderne avec électricité et écran.

Un exemple d'harmonie ludo-narrative créée par le système d'indices explicites est donné par l'entretien avec Michaël Freudenthal :

J'ai [participé à] un escape game dans lequel les joueurs ont en leur possession une tablette qui est en fait, dans l'histoire, un outil très évolué capable de scanner les environs, de détecter les anomalies et de les signaler au joueur. Étant donné que les joueurs incarnent des voyageurs temporels, il est logique qu'ils soient en possession d'une telle technologie.

Ici, le système d'indices explicites appartient à la diégèse et renforce même le récit. En effet, utiliser un objet technologique aussi avancé renforce la croyance des joueurs qu'ils sont bien des voyageurs temporels.

\section{Le maître du jeu comme PNJ}

Quand le maître du jeu est un PNJ, ce signal transmis au joueur par la jouabilité peut être difficile à justifier dans le récit : pourquoi une personne qui connaît les solutions aux énigmes et qui accepte d'aider les joueurs ne les aide-t-il que de manière ponctuelle 

peut être trouvé dans le Secret $d u$ Pirate. Les joueurs se rendent sur le bateau d'un célèbre pirate afin de suivre les indices qu'il a laissé à propos de la cachette de son trésor. En retrouvant la pierre précieuse, ils lèveront la malédiction qui pèse sur le capitaine et qui le maintient à l'état de spectre depuis trois-cents ans. Ainsi, leur mission est bénéfique pour ce dernier, qui aurait tout intérêt à les aider. C'est d'ailleurs ce qu'il fait en leur transmettant des indices par le biais de son grimoire magique. Le récit justifie le fait qu'il n'aide pas totalement les joueurs à trouver son trésor par sa nature de pirate, qui est foncièrement mauvaise. De ce fait, malgré sa volonté de briser la malédiction qui l'enchaîne à son navire depuis trois-cents ans, le capitaine refuse de guider les joueurs jusqu'à la pierre précieuse alors qu'il en connait la localisation avec précision.

Sébastien Decoster donne un exemple d'harmonie ludo-narrative liée au personnage :

J'ai joué à un escape game dans lequel tout le gameplay repose sur un téléphone public; les joueurs disposent d'un bottin rempli de numéros de téléphone. En résolvant des énigmes, les joueurs isolent des numéros particulièrement importants. Lorsque les joueurs les composent, c'est le maître du jeu qui répond. Un déformeur de voix permet au maître du jeu de donner des informations aux joueurs en interprétant des personnages. Il n'y a donc pas un, mais des dizaines de personnages qui aident les joueurs.

Dans ce cas, le personnage incarné par le maître du jeu change en fonction du numéro appelé par les joueurs. En conséquence, il est tout à fait logique que l'interlocuteur ne connaisse pas toutes les solutions aux défis.

\section{Les objets : le problème des leurres}

Les objets sont au cœur des JE. Si nous considérons leur utilité, nous pouvons les classer en deux catégories. Ils peuvent soit favoriser l'immersion du joueur en participant à la création d'un décor, soit aider la progression des joueurs ou leur permettre de résoudre les défis. L'utilité des objets est totalement indépendante de leur nature : un objet qui est traditionnellement utilisé en tant que décoration peut tout à fait être l'élément central de la résolution d'un défi dans un JE. A l'inverse, un objet que l'on pourrait considérer comme utile, par exemple un livre, peut être utilisé de façon purement décorative. Mais avant tout, les objets de décoration permettent de créer une ambiance propice à l'immersion et à la narration environnementale. La narration environnementale crée les conditions préliminaires à une expérience narrative immersive (Jenkins, 2004). Le problème est que lorsqu'il y a trop d'objets inutiles ou de leurres, le joueur risque de se sentir perdu. Il ne comprend plus l'objectif qu'il doit atteindre. Il y a donc un conflit entre l'ajout d'objets de décorations pour favoriser l'immersion du joueur, créer une narration environnementale, et le risque que les joueurs confondent les objets inutiles avec des objets essentiels pour résoudre les défis (Nicholson, 2016, p. 13).

Face à ce problème, de nombreuses salles de JE décident de poser des écriteaux « Ne pas toucher » sur les objets purement décoratifs, ou d'indiquer des limites lors du briefing, comme «ne pas toucher aux prises électriques». Cela permet de les préserver dans le cas où ils sont fragiles, mais également d'indiquer aux joueurs qu'il est inutile de se pencher davantage sur ces objets. Il s'agit donc d'une tension entre les dimensions Moteur et Diégèse. Dans le JE La Serre, les joueurs découvrent un extrait de journal dans 
lequel seules quelques lignes sont utiles à la résolution d'un défi. Pour ne pas rendre les joueurs confus, le reste de la page a été couvert avec un texte générique: "lorem ipsum... ». Il s'agit donc ici d'une tension entre les dimensions Épreuve et Diégèse. Dans les deux cas, cet élément extradiégétique entraîne une dissonance ludo-narrative chez le joueur qui peut alors, en principe, sortir de l'immersion.

Tout comme les indices explicites, les objets, qui participent à l'épreuve, peuvent ne pas être intégrés à la diégèse comme le remarque Fiona Buchou : "Nous étions censés être dans un laboratoire scientifique. A un moment, nous sommes rentrés dans une centrifugeuse. A l'intérieur, nous avons trouvé un coffre de pirates, avec un cryptex à l'intérieur".

\section{Des relations ludo narratives à un modèle d'immersion : quels effets sur le joueur?}

51 Le modèle d'Aarseth (2012) nous a été utile pour classer des exemples rapportés de dissonances ou d'harmonies ludo-narratives possibles dans le récit d'expériences de JE. Il nous faut donc nous pencher sur l'influence que ces éléments peuvent avoir sur le joueur et l'immersion dans le jeu.

\section{Dissonances ludo-narrative et suspension d'incrédulité}

Ressentir une dissonance cognitive est inconfortable pour la personne qui en fait l'expérience. En conséquence, celle-ci agit afin de réduire la dissonance. Lorsque le comportement d'une personne est dissonant par rapport à l'une de ses connaissances, elle dispose de deux moyens d'action pour réduire sa dissonance: changer son comportement ou sa connaissance. Par exemple, si une personne fume tout en sachant que fumer est nocif, elle peut réduire cette dissonance en arrêtant de fumer ou en se convaincant que fumer n'est pas nocif (Festinger, 1957).

Obliger le joueur à justifier une dissonance ludo-narrative met en danger la suspension consentie de l'incrédulité. Certes, le joueur est capable d'ignorer certaines incohérences afin de pouvoir apprécier le récit du jeu; mais si ces incohérences sont trop fréquentes ou trop fortes, cela peut briser la suspension consentie de l'incrédulité et par conséquent remet en question son engagement dans l'attitude ludique (Freudenthal, 2019) et son immersion dans le jeu (Madigan, 2010). Ainsi une étude de la rétention des joueurs d'un jeu vidéo a noté que certains joueurs mentionnent la dissonance ludo-narrative comme raison de leur baisse d'intérêt (Strååt et Verhagen, 2018).

54 Toutefois, si cet effet peut être involontaire, il peut au contraire être créé avec le dessein d'amener le joueur à prendre du recul sur la situation sans forcément se désengager complètement, d'une façon comparable à l'effet de distanciation du théâtre de Brecht (Pötzsch, 2017 ; Levin, 2020). Ainsi, les moments où le joueur lit un panneau « ne pas toucher » et l'ignore ne l'amènent pas forcément à se désengager. 


\section{Exemples rapportés de sortie de l'immersion}

Alors que nous avons pu identifier des cas de dissonance ludo-narrative, nous avons peu d'indications sur les effets de celles-ci sur les joueurs. Au sujet du cryptex trouvé dans un laboratoire scientifique Fiona Buchou note «Les deux objets ne collaient pas ensemble mais surtout ils ne correspondaient pas à la pièce dans laquelle nous nous trouvions. Cela nous a beaucoup sortis de l'immersion ».

Le sujet est aussi abordé de manière indirecte par Alexandre Carturan quand il explique pourquoi il n'utilise jamais de panneau « Ne pas toucher » en tant que concepteur :

Nous n'avons jamais utilisé d'autocollants disant aux joueurs de ne pas toucher un objet. C'est la pire chose qui fait se rendre compte au joueur qu'il n'est pas dans le monde dans lequel l'escape game l'a plongé. Pour éviter ces autocollants, j'ai déjà caché des câbles dans des cordes de marin dans le cadre d'un escape game sur le thème des pirates.

Nous trouvons des corrélations entre sortie de l'immersion, au sens d'un désengagement plus ou moins grand, et ce que nous décrivons comme dissonance ludonarrative. S'il est possible que le joueur trouve des façons de maintenir son sentiment d'harmonie ludo-narrative, en ignorant des contradictions ou en prenant du recul, il peut toutefois vivre une dissonance comme un endommagement de son expérience de jeu. C'est ce qui amène certains JE à préférer cacher les éléments méta-diégétiques au profit d'un décor total, sans câble ou écriteau.

\section{Conclusion et perspectives}

modèle d'Aarseth permet d'identifier les éléments du jeu contribuant à la dissonance ou l'harmonie ludo-narratives des JE. Le modèle MEDIAL, lui, nous a permis d'identifier les dimensions de l'engagement du joueur liés à ces cas de dissonance et d'harmonie ludo-narratives. On retrouve ces variations au travers des tensions et des interdépendances entre les différentes dimensions de l'engagement, qui une fois identifiées clarifient l'expérience rapportée par les joueurs et certains choix de conception des JE.

59 Nous proposons donc ici une extrapolation du modèle MEDIAL qui prend en compte la nature des interactions entre les dimensions, qui peuvent se faire en harmonie ou en contradiction. Il pourrait être pertinent d'utiliser également à l'avenir le modèle GFI (Cardona-Rivera et al., 2020), qui peut compléter ces deux modèles en apportant les notions d'objectifs («goals») et de rétroaction ( «feedback») en relation au jeu et à l'interprétation réalisée par le joueur. Les notions d'objectif, de rétroaction et d'interprétation sont en effet directement liées aux observations faites respectivement au sujet des événements, du système d'aide et des objets.

Il reste toutefois l'idée, soulignée par deux personnes interrogées, que la présence de dissonances ludo-narratives contribue à l'immersion. Le corpus d'entretiens de Nugues (2019) n'est pas suffisant pour entretenir cette idée, mais est suffisant pour ouvrir la voie à des questionnements nuancés sur l'engagement qui accepterait sa variation d'intensité et de dimension comme part intégrante de l'expérience de JE, voire de jeu. 


\section{BIBLIOGRAPHIE}

AARSETH E. (2012). «A narrative theory of games ». Proceedings of the International Conference on the Foundations of Digital Games, pp. 129-133.

ADAMS E. (1999). The Designer's Notebook: Three Problems for Interactive Storytellers. Gamasutra. https://www.gamasutra.com/view/feature/131821/the_designers_notebook_three_.php

ARSENAULT D. et PICARD M. (2008). « Le jeu vidéo entre dépendance et plaisir immersif : Les trois formes d'immersion vidéoludique ». Proceedings of HomoLudens : Le jeu vidéo : un phénomène social massivement pratiqué, pp. 1-16.

BLUMENTHAL V. (2020). «Consumer immersion in managed visitor attractions: The role of individual responses and antecedent factors ». Scandinavian Journal of Hospitality and Tourism, 20(1), pp. 4-27.

BLUMENTHAL V. et JENSEN Ø. (2019). « Consumer immersion in the experiencescape of managed visitor attractions: The nature of the immersion process and the role of involvement ». Tourism Management Perspectives, 30, pp. 159-170.

BONENFANT M., et PHILIPETTE T. (2018). « Rhétorique de l'engagement ludique dans des dispositifs de ludification ». Sciences du jeu, 10, http://journals.openedition.org/sdj/1422.

BROUGÈRE G. (2005). Jouer/Apprendre, Paris, Economica.

CAÏRA O. (2018). « Les dimensions multiples de l'engagement ludique ». Sciences du jeu, 10, http:// journals.openedition.org/sdj/1149.

CALLEJA G. (2011). In-Game: From Immersion to Incorporation, Cambridge, MIT Press.

CARDONA-RIVERA R. E., ZAGAL J. P. et DEBUS M. S. (2020). « Narrative Goals in Games: A Novel Nexus of Story and Gameplay ». International Conference on the Foundations of Digital Games, pp. 1-4.

CLARE A., et CHOULDHURY S. (2016). Escape the Game: How to Make Puzzles and Escape Rooms. Scotts Valley (CA), CreateSpace Independent Publishing Platform.

DUNNE D. (2014). « Multimodality or Ludo-Narrative Dissonance: Duality of Presentation in Fringe Media ». Proceedings of the 2014 Conference on Interactive Entertainment, pp. 1-4.

ERMI L., et MÄYRÄ F. (2005). « Fundamental components of the gameplay experience: Analysing immersion ", in S. D. Castell et J. Jenson (Eds.), Worlds in Play: International Perspectives on Digital Games Research), Berne, Peter Lang, pp. 37-53.

FESTINGER L. (1957). A Theory of Cognitive Dissonance. Palo Alto, Stanford University Press.

FRENCH S., MARMOR SHAW J. (2015). The unbelievably lucrative business of escape roomsMarketWatch. https://www.marketwatch.com/story/the-weird-new-world-of-escape-roombusinesses-2015-07-20

FREUDENTHAL M. (2019). « Vers une expérience holistique. », in E. Lebret, C. Quesne (dir.) L'escape game, une pratique pédagogique innovante. Futuroscope, Editions Canopé, pp. 21-22. GENETTE G. (1972). Figures III. Paris, Seuil.

GENVO S. (2020). « Faire jouer la vie d'autrui. Approche ludonarrative du jeu expressif autobiographique Lie in my heart ». Cahiers de Narratologie. Analyse et théorie narratives, 38. https://journals.openedition.org/narratologie/11711 
GRANDJEAN G. (2018). « Quel lieu pour l'exploration? Approche formelle d'une incertitude. » communication au colloque Entre le jeu et le joueur : écarts et médiations, Liège, http:// www.expressivegame.com/publications/colloques/quel-lieu-pour-lexploration-approcheformelle-dune-incertitude-spatialisee-g-grandjean/

GRANDJEAN G. (2020). Le langage du level design : Analyse communicationnelle des structures et instances de médiation spatiales dans la série The Legend of Zelda (1986-2017), thèse de doctorat, Université de Lorraine.

HOCKING C. (2009). «Ludonarrative dissonance in Bioshock: The problem of what the game is about », Well Played, 1, pp. 255-260.

HOU H.-T., CHOU Y.-S. (2012). « Exploring the Technology Acceptance and Flow State of a Chamber Escape Game-Escape the Lab for Learning Electromagnet Concept ». Work-In-Progress Poster (WIPP). Proceedings of $20^{\text {th }}$ International Conference on Computers in Education, Singapore, pp. 38-41

HUIZINGA J. (1951 [1938]). Homo Ludens, essai sur la fonction sociale du jeu. Paris, Gallimard.

JENKINS H. (2004). « Game Design as Narrative Architecture » Electronic book review. http:// electronicbookreview.com/essay/game-design-as-narrative-architecture/

KAPP S. (2013). « L'immersion fictionnelle collaborative. Une étude de la posture d'engagement dans les jeux de rôles grandeur nature ». Thèse de doctorat, Ecole des Hautes Etudes en Sciences Sociales (EHESS) ; Université Libre de Bruxelles.

KARHULAHTI V.-M. (2012). « Suspending Virtual Disbelief: A Perspective on Narrative Coherence », in D. Oyarzun, F. Peinado, R. M. Young, A. Elizalde et G. Méndez (eds.), Interactive Storytelling, New York, Springer, pp. 1-17

KOENITZ H. (2018). « Narrative in Video Games », in N. Lee (ed.), Encyclopedia of Computer Graphics and Games, New York, Springer International Publishing, pp. 1-9.

KOLAR T. et ČATER B. (2018). « Managing group flow experiences in escape rooms ». International Journal of Contemporary Hospitality Management, 30(7), pp. 2637-2661.

LEVIN H. (2020). « Metareflection », in E. Saitta, J. Koljonen, J. Särkijärvi, A. Serup Grove, P. Männistö et M. Makkonen (eds.), What Do We Do When We Play?, pp. 62-74.

MADIGAN J. (2010). « The Psychology of Immersion in Video Games ». The Psychology of Video Games. https://www.psychologyofgames.com/2010/07/the-psychology-of-immersion-in-videogames/

NICHOLSON S. (2016). « Ask Why: Creating a Better Player Experience through Environmental Storytelling and Consistency in Escape Room Design ». Meaningful Play, pp. 1-17.

NUGUE A. (2019). Les relations ludo-narratives dans les escape games. Thèse professionnelle, Cnam Enjmin, Gobelins école de l'image.

PÖTZSCH H. (2017). « Playing Games with Shklovsky, Brecht, and Boal: Ostranenie, V-Effect, and Spect-Actors as Analytical Tools for Game Studies ». Game Studies, 17(2). http://gamestudies.org/ 1702/articles/potzsch

PYNENBURG T. (2012). « Games Worth a Thousand Words: Critical Approaches and Ludonarrative Harmony in Interactive Narratives ». Honors Theses and Capstones. 70, University of New Hampshire. https://scholars.unh.edu/honors/70 
SALEN K. et ZIMMERMAN E. (2003). « This is not a game: Play in cultural environments ». Proceedings of the 2003 DiGRA International Conference: Level Up, vol. 2. http://www.digra.org/wpcontent/uploads/digital-library/05164.10000.pdf

STASIAK A. (2016). «Escape Rooms: A New Offer in the Recreation Sector in Poland." Turyzm, 26(1), pp. 31-47.

STRÅÅT B. et VERHAGEN H. (2018). « Exploring Video Game Design and Player Retention- a Longitudinal Case Study ». Proceedings of the $22^{\text {nd }}$ International Academic Mindtrek Conference, pp. 39-48.

TOH W. (2015). A multimodal discourse analysis of video games: A ludonarrative model, Thèse de doctorat, National University of Singapore

TRÉPANIER-JOBIN G. et COUTURIER A. (2018). « L'immersion fictionnelle au-delà de la narrativité ». Sciences du jeu, 9, http://journals.openedition.org/sdj/950.

WIEMKER M., ELUMIR E. et CLARE A. (2005). « Escape Room Design as a Game-Based Learning Process for STEAM Education ", $13^{\text {th }}$ European Conference on Games Based Learning, Odense, Danemark, pp. 55-75.

WILLIAMS M. K., et MASCIONI M. M. (2014). The Out-of-Home Immersive Entertainment Frontier: Expanding Interactive Boundaries in Leisure Facilities. London, Routledge.

\section{NOTES}

1. Nous remercions Charles Ayats, Jeremy Ha, Aurélien Lefrançois, Zoé Picardat pour les discussions autour du sujet de cet article.

2. Il est à noter que seule la version PDF de l'article indique une interdépendance des flèches, cette mention est absente sur la version en ligne. Nous choisissons de la prendre en considération.

\section{RÉSUMÉS}

Les jeux d'évasion ont rencontré un grand succès ces dernières années avec une tendance à l'augmentation des aspects narratifs. Alors que l'immersion est présentée comme une caractéristique saillante de cette expérience ludique, il n'existe pas de modèle permettant d'analyser l'immersion d'un joueur dans un jeu d'évasion. Pourtant, les relations entre le récit et la jouabilité d'un jeu pouvant influer sur l'engagement des joueurs viennent renforcer ou réduire l'immersion. Nous proposons de combiner le modèle d'analyse narrative d'Aarseth pour identifier des éléments du jeu contribuant à la dissonance ou l'harmonie ludo-narratives des jeux d'évasion et le modèle MEDIAL pour identifier les dimensions de l'engagement du joueur liées à ces cas de dissonance et d'harmonie ludo-narratives.

Escape games have become very popular in recent years, with a growing focus on their narrative aspects. While immersion is presented as a salient feature of this game experience, there is a lack of models to analyze the immersion of players in an escape game. Yet, the relationship between 
narration and gameplay can influence players' engagement and the feeling of immersion. We propose to combine Aarseth's narrative analysis model to identify elements of the game contributing to ludo-narrative dissonance or harmony in escape games, and the MEDIAL model to identify the resulting dimensions of players' engagement.

\section{INDEX}

Mots-clés : jeu d'évasion, immersion, engagement, flow, dissonance ludo-narrative, harmonie ludo-narrative

Keywords : escape game, immersion, engagement, flow, ludo-narrative dissonance, ludonarrative harmony

\section{AUTEURS}

\section{ANTOINE TALY}

Laboratoire de Biochimie Théorique, Institut de Biologie Physico-Chimique

\section{ANNELYSE NUGUE}

Conceptrice de jeux

\section{MICHAEL FREUDENTHAL}

Experice, Université Sorbonne Paris Nord 